\title{
Ordered Arrangement in Binary Mixture of Polymer Microspheres
}

\author{
Kazuroh Tokushige, Kohei Kawahara, ${ }^{\dagger}$ and Takashi Fukutomi \\ Department of Polymer Chemistry, Tokyo Institute of Technology, \\ 2-12, Ookayama, Meguro-ku, Tokyo 152, Japan
}

(Received December 18, 1995)

\begin{abstract}
The formation of ordered arrangement of binary microsphere (or microgel) system with different compositions but same diameter was investigated by computer simulation and experiment. As a result of simulation, alternately arranged structure of binary particles was obtained under the condition that repulsion between particles of different composition was weaker than that between particles of the same composition. In the experiment, the mixtures of poly(4-vinylpyridine) particles (S-4VP) quaternized at various degree and poly(styrene-co-hydroxyethyl methacrylate) particles (S-St-HEMA) were used. Ordered layer arrangement of S-4VP appeared in closed packed S-4VP/S-St-HEMA structure at highly quaternized degree of S-4VP.

KEY WORDS Microgel / Microsphere / Arrangement / Binary Mixture / Computer Simulation / Interparticle Contact Angle /
\end{abstract}

Microspheres (or Microgels) with narrow size distribution form ordered structure. In homogeneous system the structure is face centered cubic (FCC) structure. ${ }^{1-3}$ And the arrangement of binary mixture has been reported in detail, when binary particles have difference between their diameters only. Sanderes found out that the iridescence of Brazil opal arises from ordered arrangement of binary silica particles with different diameters. ${ }^{4}$ In liquid state, Hachisu et al. observed binary mixture of polystyrene particles with different diameters. They found several alloy structure, and the structure was the closest packing of component particles. ${ }^{5,6}$ They found out that the structure was affected only by the ratio of two component particles. ${ }^{6}$

Recently $\mathrm{MgCu}_{2}$ and $\mathrm{NaCl}$ types ordered structure were found in the mixture of poly(4-vinylpyridine) particles (S-4VP) and polystyrene particles with - $\mathrm{OH}$ groups on their surface (polystyrene/hydroxyethylmethacrylate: S-St-HEMA). ${ }^{7,8}$ This binary system was composed of particles with different diameters and different composition. The structure was that corresponded to the closest packed structure. The effect of the diameter ratio was the same as that in mixture of binary microsphere with the same composition. Geometrical factor seems to affect the arrangement in these cases. Ordered arrangement was also observed when S-4VP and S-St-HEMA particles have the same diameters. The structure was identified as FCC (same as the closest packing) as a whole. But each individual species located in a disorderly manner. The binary system, composed of particles with same diameter is more complicated than that composed of particles with different diameters, because the difference of interparticle interactions affects directly on the arrangement.

In this study, computer simulation for mixture of binary microgels was carried out. Then two kinds of particles: partially quaternized S-4VP and St-HEMA were synthesized and mixed actually at mixing ratio $1: 1$.

\section{SIMULATION}

Simulation was carried out by molecular dynamics method with an NEC PC-9821As personal computer. By this method inter particle interaction was calculated for each pair of particles, and then all particles were made to move at a time. To simplify the system, the diameters of binary particles are assumed to be the same. Interactions were changed for the pair of different particles. Interparticle interactions affected by their composition is unclear. But in the liquid state, solvation should be one of the most important factor stabilizing the dispersion. In solid state, particles contact with each other (Figure 1(a)). If certain solvent is added and particles prefer to contact with the solvent molecules, particles will disperse into the solvent (liquid state,
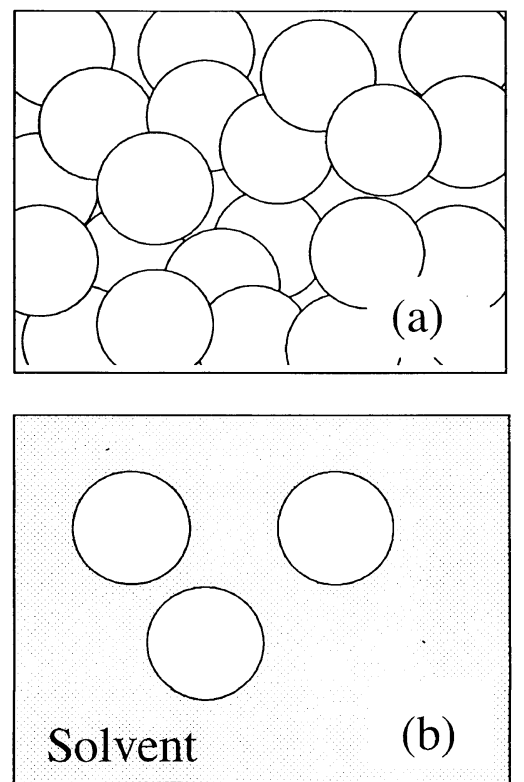

Figure 1. (a) Solid state: particles contact with each other. (b) Liquid state: particles contact with solvent on their surface affinity of particle-solvent $>$ particle-particle.

\footnotetext{
${ }^{\dagger}$ Present address: Japan Advanced Institute of Science and Technology, 15, Asahi-dai, Tatsunokuchi-cho, Ishikawa, 923-12, Japan.
} 
Figure 1(b)). Comparing these two states, the affinity of particle-solvent is higher than that of particle-particle. So the difference of affinity with solvent can be regarded as an apparent repulsive force between particles. When dispersion contains binary particles, the affinity of particle-solvent and particle-particle will vary with the composition of particles and solvent. This kind of interaction arising from the affinity, is expected to be analogous to van der Waals potential. For simplification, interparticle interaction was calculated by modifying van der Waals potential as following:

$$
V_{\mathrm{R}}=k\left\{2 a^{2} /\left(r^{2}-4 a^{2}\right)+2 a^{2} / r^{2}+\ln \left(1-4 a^{2} / r^{2}\right)\right\}
$$

where $a$ is radius of particle, $r$ is distance between particle centers, and the value of $k$ varies from zero to unity. If $k<0$ (i.e., interaction between different particles is attractive force) particles will aggregate rapidly. And if $k>1$, different particles will not be mixed each other. So the $k$ values of $k>1$ and $0<k<1$ were excluded.

To evaluate the regularity of arrangement, radial distribution function $(g(r))$ and distribution of inter particles contact angle (ICA) $)^{7,9}$ was calculated. $g(r)$ gives the probabirity of finding the center of a particle at a distance $(r)$ from the center of a reference particle. It can be writtern

$$
g(r)=N_{\mathrm{p}}(r) / N_{\mathrm{p}}
$$

where $N_{\mathrm{p}}$ is the average number of particles per unit volume, and $N_{\mathrm{p}}(r)$ is the radial distribution of particle density. $G(r)$ was calculated for pairs of the same particles $(\mathrm{A}-\mathrm{A}$ and $\mathrm{B}-\mathrm{B})$, and different particles $(\mathrm{A}-\mathrm{B})$.

ICA (angle composed of three neighbouring three particles $\mathrm{P}, \mathrm{Q}$, and $\mathrm{R}$; $\mathrm{PQR}$ ) distribution should have peaks at particular angles in ordered arrangements. ICA was calculated for the sets of particles $\mathrm{A}-\mathrm{A}-\mathrm{A}$, B-B-B, A-B-A, and B-A-B (Figure 2).

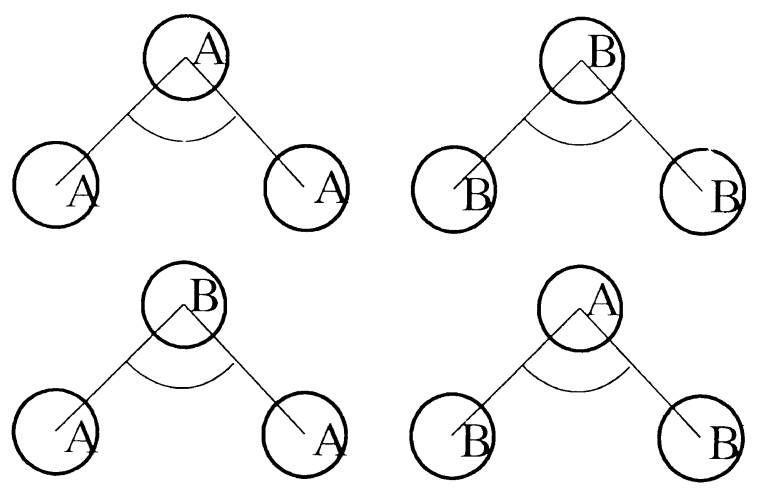

Figure 2. Four kinds of interparticle contact angle distribution (ICA).

\section{EXPERIMENTAL}

\section{Materials and Preparation of Films}

Many S-4VP samples was synthesized varying the volume ratio of $4 \mathrm{VP}$ and $\mathrm{H}_{2} \mathrm{O} .^{7,10} \mathrm{~S}$-St-HEMA samples were synthesized by surfactant-free emulsion polymerization also varying the volume ratio of St and HEMA. The polymerization was carried out in deionized water for $8 \mathrm{~h}$ with V-50 (2,2'-Azobis(2-methylpropionamidine)dihydrochloride) as initiator. The recipes and their diameters are shown in Table I. Among these samples, a pair with almost same diameter were selected. The SEM micrographs of selected samples are shown in Figure 3. 4VP particles have interparticle crosslinking to prevent deformation and dissolution. Contrary to this, S-StHEMA particles have no crosslinking. They are able to dissolve easily in good solvents, for example dioxan, acetone and the mixture of these solvents with $\mathrm{H}_{2} \mathrm{O}$. Particles were purified by filtration and washing with deionized water and methanol. Then S-4VP particles were partially quaternized with $\mathrm{CH}_{3} \mathrm{I}$ in ethanol for 48 hours at room temperature and then for 12 hours at $60^{\circ} \mathrm{C}$. Under these conditions, quaternization was almost stoichiometric. The quaternization degree was adjusted to $0 \%, 25 \%, 50 \%$, and $75 \%$ of overall pyridine units. $\mathrm{S}-4 \mathrm{VP}$ emulsions were mixed with S-St-HEMA emulsions at mixing ratio 1 (solid weight): 1 (solid weight). To obtain films of the binary mixture, dispersion was casted on tefrom sheet, and slowly dried at $5^{\circ} \mathrm{C}$.

\section{Observation of Binary Mixture}

Arrangement of particle was observed with scanning electron microscope (SEM). When diameters of mixing particles are almost the same, one cannot distinguish the two kinds of particles with SEM. To distinguish St-HEMA particles and 4VP particles, the mixture was treated as following. S-4VP particles were crosslinked mutually with saturated dibromobutane gas at room for 3 days. Then St-HEMA particles were extracted by dioxane (good solvent of polystyrene) for 48 hours at room temperature. S-St-HEMA microspheres were easily extracted. By this procedure, one could observe only S-4VP particles and their arrangement clearly with SEM. The positions of S-St-HEMA are recognized as voids of specimens.

The regularity of arrangement was evaluated by ICA distribution. To obtain ICA, it is necessary to calculate the coordinates of individual particles from SEM micrographs. The detailed method of calculation is as follows: first, two SEM micrographs of the same part were taken at different angles around one rotating axis.

\begin{tabular}{|c|c|c|c|c|c|}
\hline \multirow{2}{*}{$\begin{array}{l}\text { Sample } \\
\text { code }\end{array}$} & \multicolumn{4}{|c|}{ Feed Conditions } & \multirow{2}{*}{$\frac{\text { Diameter }^{\mathrm{a}}}{\mathrm{nm}}$} \\
\hline & Monomer & $\begin{array}{l}\text { Concn } \\
\mathrm{wt} \%\end{array}$ & $\begin{array}{c}\mathrm{DVB}^{\mathrm{b}} / \text { Monomer } \\
\mathrm{mol} / \mathrm{mol}\end{array}$ & $\frac{\mathrm{HEMA}^{\mathrm{c}} / \mathrm{St}}{\mathrm{mol} / \mathrm{mol}}$ & \\
\hline S-4VP & 4-Vinylpirydine & 2 & 0.200 & - & 240 \\
\hline S-St-HEMA & Styrene & 15 & - & 0.05 & 240 \\
\hline
\end{tabular}

Table I. Preparative conditions of 4VP microgel and St-HEMA microsphere

${ }^{\mathrm{a}}$ Number average diameter determined by scanning electron micrograph. ${ }^{\mathrm{b}}$ Divinylbenzene. ${ }^{\mathrm{c}}$ Hydroxyethylmethacrylate. Initiator: V-50 2,2-azobis(2-methylpropionamidine)dihydro-chloride; V-50/monomer (wt/wt) $=0.02$. Conditions: $70^{\circ} \mathrm{C}, 8 \mathrm{~h}$ under $\mathrm{N}_{2}$ atmosphere. 


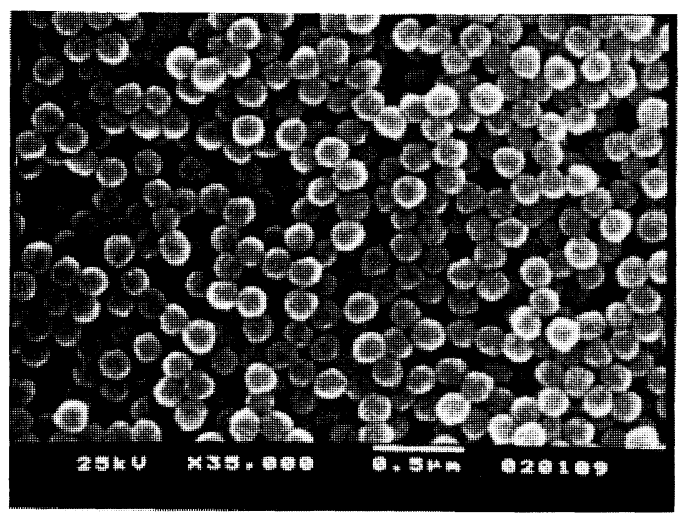

(a)

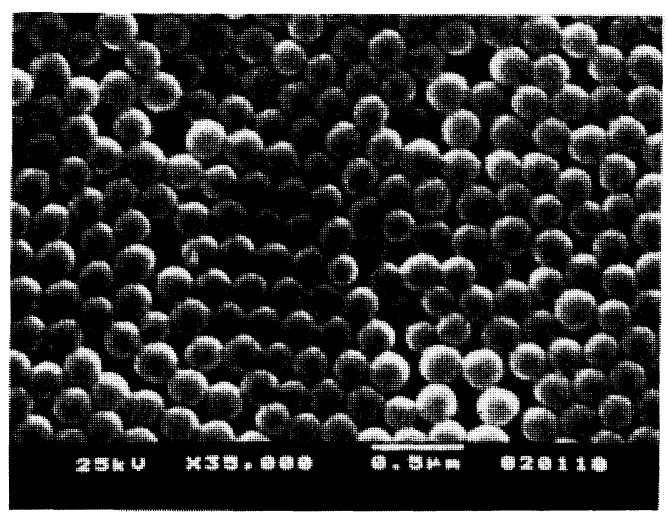

(b)

Figure 3. SEM micrograph of (a) 4VP microgels, and (b) St-HEMA microspheres.

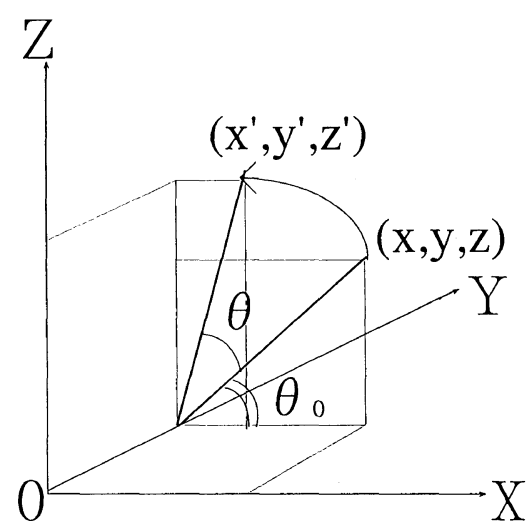

Figure 4. The calculation method of coordinates of a particle.

This rotating axis was taken as $Y$ coordinate axis as shown in Figure 4 . The $X$ and $Z$ coordinate axes were taken as shown in the same figure. If coordinates of a particle before and after rotation (with angle $\theta$ ) are expressed by $(x, y, z)$ and $\left(x^{\prime}, y^{\prime}, z^{\prime}\right)$ respectively, the folowing relations can be derived.

$$
\begin{aligned}
& x^{\prime}=\sqrt{x^{2}+z^{2}} \cos \left(\theta+\theta_{0}\right) \\
& y^{\prime}=y \\
& z^{\prime}=\sqrt{x^{2}+z^{2}} \sin \left(\theta+\theta_{0}\right)
\end{aligned}
$$

where $\theta_{0}=\tan ^{-1}(z / x)$, and $x, y, x^{\prime}, y^{\prime}$ can be obtained directly from the SEM micrographs before and after rotation. Then the $z$ and $z^{\prime}$ can be expressed as follows:

$$
\begin{aligned}
z & =\left(x \cos \theta-x^{\prime}\right) / \sin \theta \\
z^{\prime} & =\left(x-x^{\prime} \cos \theta\right) / \sin \theta
\end{aligned}
$$

\section{RESULTS AND DISCUSSION}

\section{Simulation}

Ordered arrangement of particles appeared in the simulation cell. Figure 5 shows the arrangement looked at two different angle. Its structure was alternately arranged layer of binary particles. Radial distribution $(g(r))$ is shown in Figure 6. $g(r)$ was calculated between particles of the same kind (Figure 6(b) and (c)), between particles of different kind (Figure 6(d)), and overall particles (Figure 6(a)). They are apparently different from
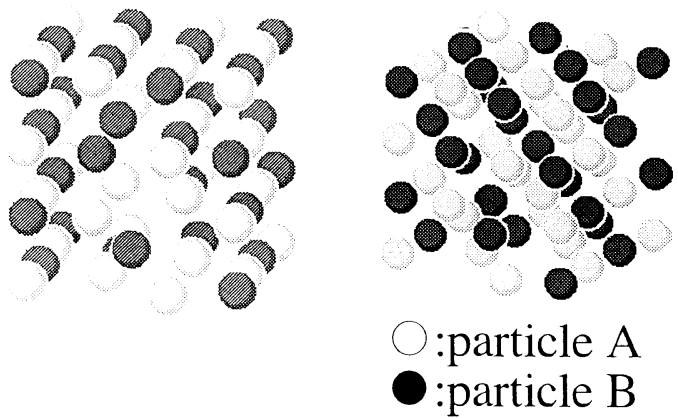

Figure 5. Ordered arrangement formed in the simulation cell (viewed from two different angle).

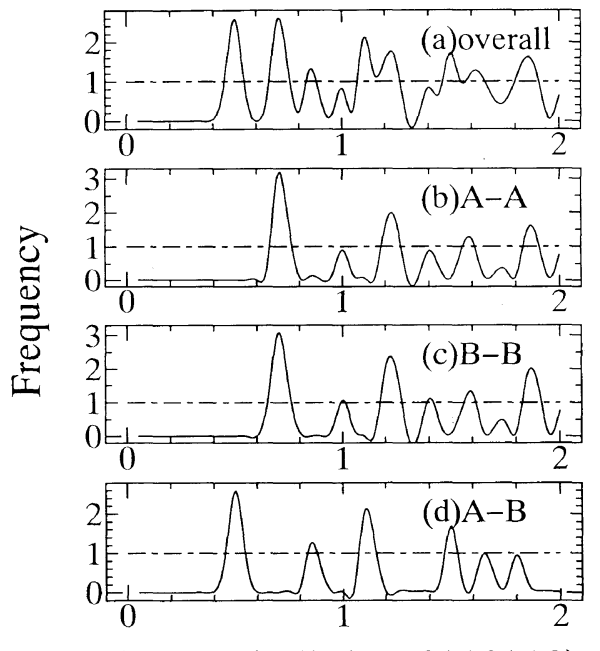

Distance $($ cell size $=2 \times 2 \times 2)$

Figure 6. Radial distribution function $(g(r))$ in the simulation cell: (a) overall; (b) particle A-A; (c) particle B-B; (d) particle A-B, B-A.

each other. If there is no difference in the arrangement of the same kind of particles, $g(r)$ will not vary with the kind of particles. These distributions show clearly that the nearest neighbour particle (the nearest peak of $g(r)$ ) is different kind. The regularity of the arrangement can be seen in ICA distribution (Figure 7). From $g(r)$ and ICA distribution, the structure is considered as $\mathrm{NaCl}$ structure (Figure 8). The ratio of peak position for first three peaks is $1: 1.44: 1.77$. This is close to that of $\mathrm{NaCl}$ structure $(1: 1.41: 1.73)$. And ICA distribution accords well with that of $\mathrm{NaCl}$ structure (Figure 8). 


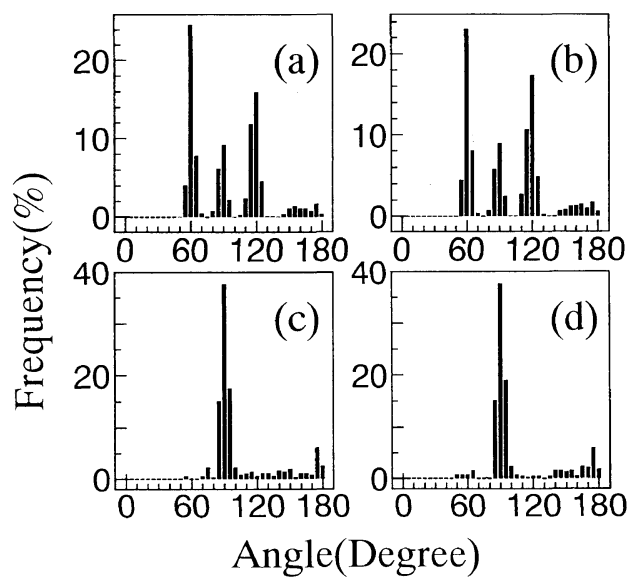

Figure 7. Interparticle contact angle distribution: (a) $\mathrm{A}-\mathrm{A}-\mathrm{A}$; (b) B-B-B; (c) A-B-A; (d) B-A-B.
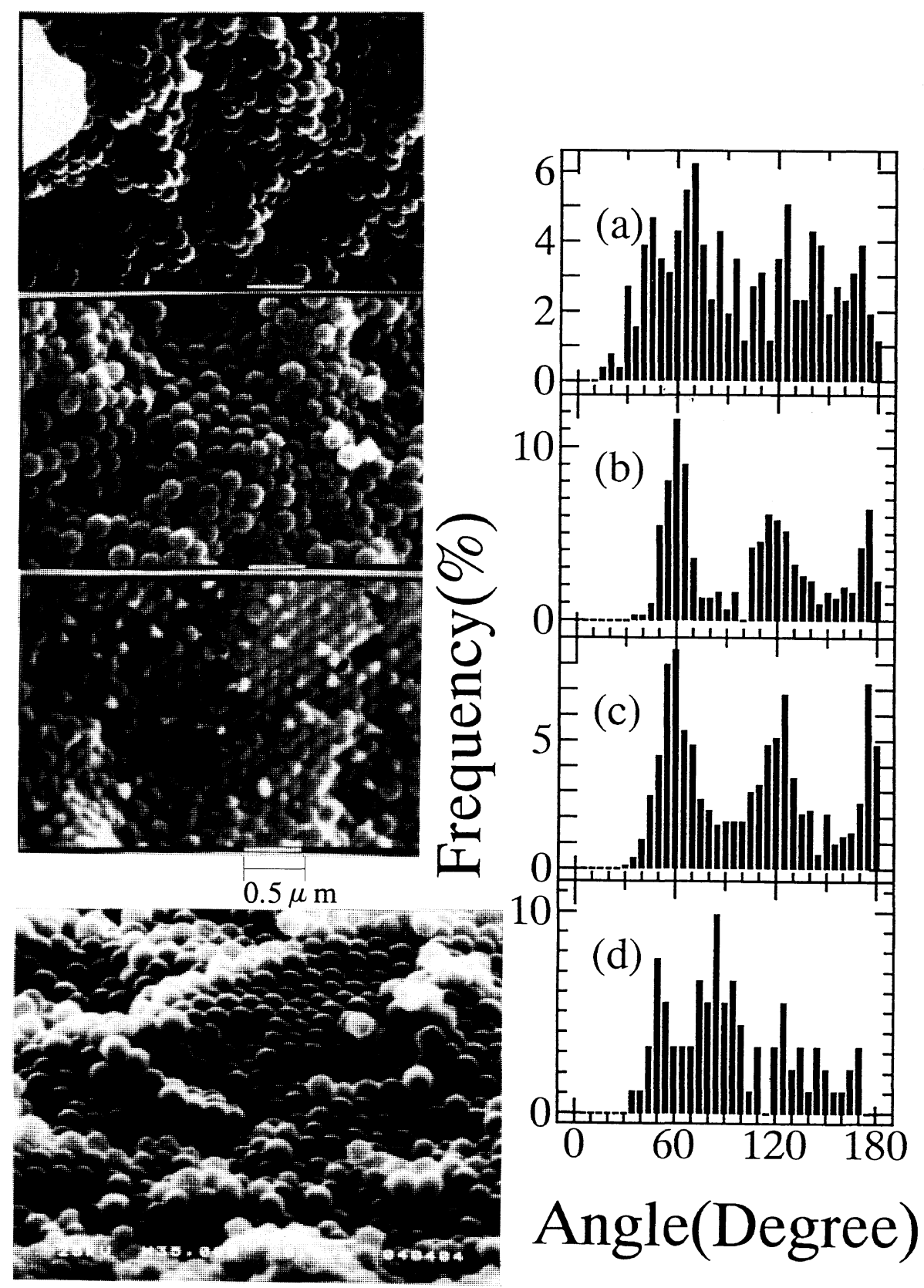

Figure 9. SEM micrographs of cross-section with their ICA distribution of binary mixture (partially quaternized 4VP microgels and St-HEMA microspheres). Quaternization degree of $4 \mathrm{VP}$ is (a) $0 \%$, (b) $25 \%$, (c) $50 \%$, and (d) $75 \%$. $1-1-1,2-2-2$; (b) particle $1-2-1,2-1-2$. 

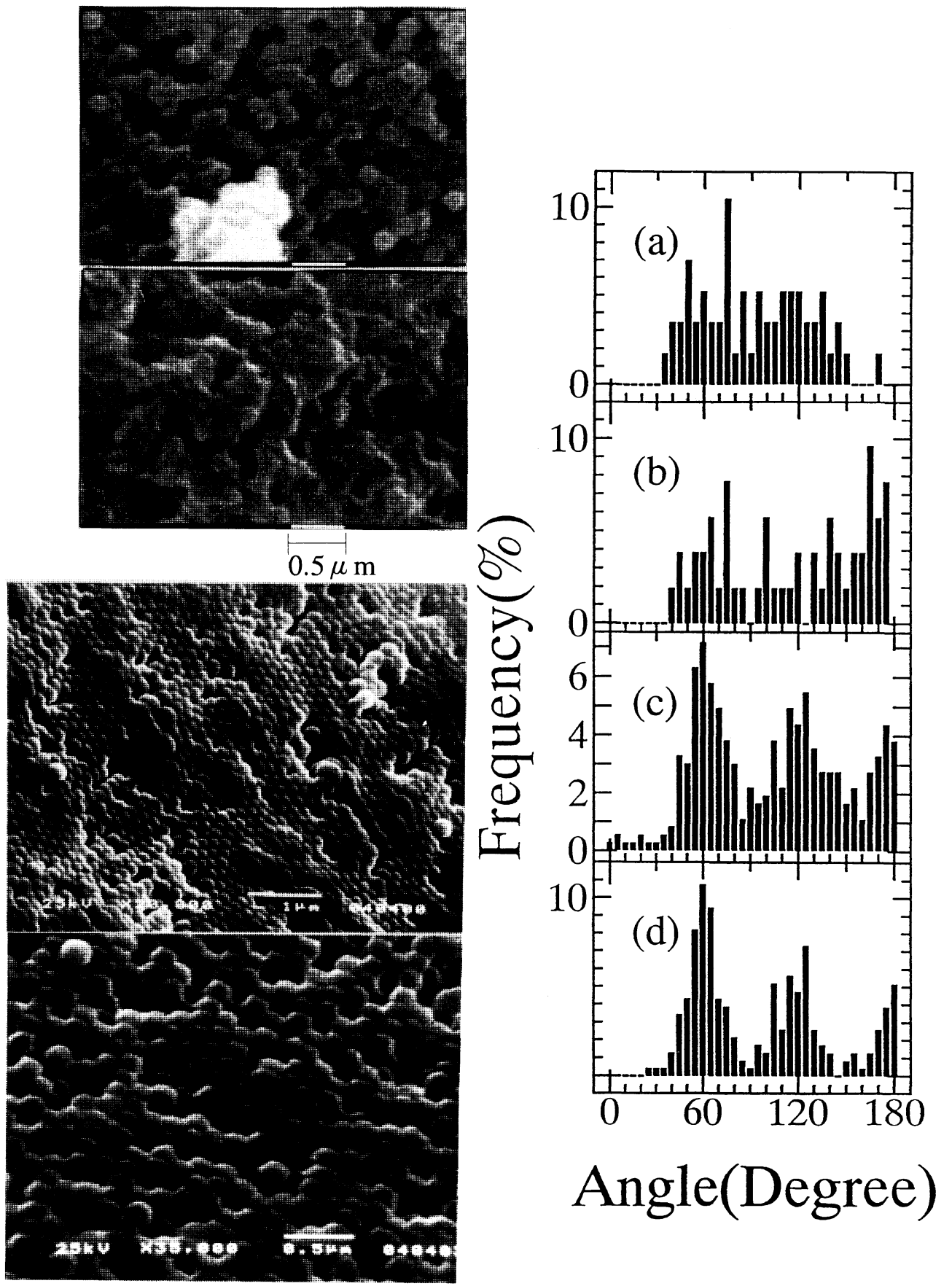

Figure 10. SEM micrographs of cross-section with their ICA distribution of binary mixture (partially quaternized 4VP microgels and St-HEMA microspheres) after extracting St-HEMA. Quaternization degree of $4 \mathrm{VP}$ is (a) $0 \%$, (b) $25 \%$, (c) $50 \%$, and (d) $75 \%$.

This result suggests that difference of interparticle interaction is one of the main factors to form regularly arranged structure. And formation of ordered structure is expected when repulsion between particles of different kind is weaker than that between particles of the same kind.

\section{Experimental}

SEM micrographs and ICA distributions of crosssection of the mixtures are shown in Figure 9. clusters where the particles form ordered arrangement (encircled by dotted line) could be observed in the mixtures (b), (c), and (d) (ratios of quaternization are $25 \%, 50 \%$, and $75 \%$ respectively). Sharp peaks corresponding to FCC structure (the closest packed one) appeared at angle $60^{\circ}$ $\left(90^{\circ}\right)$, and $120^{\circ}$ in ICA distributions of these clusters. It seems that region of ordered arrangement becomes larger, as quaternization degree of S-4VP particles increased.

The mixed S-4VP and S-St-HEMA have almost the same diameters. So one cannot distinguish the two kind of particles in mixed film. But S-St-HEMA is not crossliked in its inside, then they can easily extracted by its good solvent. The arrangement composed of only S-4VP can be observed after extracting S-St-HEMA. And the positions of S-St-HEMA are recognized as voids of specimens. Figure 10 shows SEM micrographs of crosssection of the mixture after extracting S-St-HEMA particles. Regularity was not recognized for the mixture (a) and (b) (ratios of quaternization are $0 \%$ and $25 \%$ ). 
This result shows that there exist no regularity in the arrangement of each kind of particles (S-4VP and $\mathrm{S}$-St-HEMA) in the mixture (a) and (b). But S-4VP clusters, where S-4VP particles regularly arranged, exist in the mixture (c) and (d). In ICA distribution, sharp peaks also appeared in these specimens. This result shows that the mixture of highly quaternized S-4VP (mixture (c) and (d)), arranged regularly, that is to say, S-StHEMA also arranged regularly. Considering that the position of St-HEMA particles are recognized as voids of specimen, the clusters of two kinds of particles seem to be layers.

\section{CONCLUSIONS}

Simulation was carried out for binary mixture of microgels. Alternately arranged layer structure of binary particles appeared under the condition that the repulsion between different kind of particles was weaker than the same kind of particles.

In the experiment by using $1: 1$ mixture of partially quaternized S-4VP microgels and St-HEMA microsphere, there appeared regularly arranged structure with highly quaternized S-4VP. The ordered phase increased and enlarged, as the quaternization degree of S-4VP increased. Furthermore, in these ordered phase, S-4VP themselves regularly arranged. The results of simulation and experiment accord well qualitatively.

\section{REFERENCES}

1. S. Hachisu, Hyomen, 14, 15 (1976).

2. S. Hachisu, Y. Kobayashi, and A. Kose, J. Colloid. Interface Sci., 42, 342 (1973).

3. A. Kose, M. Ozaki, Y. Kobayashi, K. Takano, and S. Hachisu, J. Colloid. Interface Sci., 44, 330 (1973).

4. J. Sanders and M. J. Murray, Nature (London), 275, 201 (1978).

5. S. Hachisu and S. Yoshimura, Nature (London), 283, 188 (1980).

6. S. Hachisu and S. Yoshimura, Hyomen, 21, 247 (1983).

7. G. H. Ma and T. Fukutomi, Trends in Polym. Sci., 3, 37 (1993).

8. G. H. Ma and T. Fukutomi, Polym. International, 30, 533 (1993).

9. G. H. Ma and T. Fukutomi, J. Appl. Polym. Sci., 43, 1451 (1991). 